\title{
Diagnosis of Pulmonary Arterial Hypertension in Children by Using Cardiac Computed Tomography
}

Shyh-Jye Chen, MD, PhD ${ }^{1}$, Jou-Hsuan Huang, MD ${ }^{1}$, Wen-Jeng Lee, MD, PhD ${ }^{1}$, Ming-Tai Lin, MD, PhD ${ }^{2}$, Yih-Sharng Chen, MD, PhD ${ }^{3}$, Jou-Kou Wang, MD, PhD²

Departments of ${ }^{1}$ Radiology and Medical Imaging, ${ }^{2}$ Pediatrics, and ${ }^{3}$ Surgery, National Taiwan University Hospital, Medical College, National Taiwan University, Taipei, Taiwan

Objective: To establish diagnostic criteria for pulmonary arterial hypertension (PAH) in children by using parameters obtained through noninvasive cardiac computed tomography (CCT).

Materials and Methods: We retrospectively measured parameters from CCT images of children from a single institution in a multiple stepwise process. A total of 208 children with mean age of 10.5 years (range: 4 days-18.9 years) were assessed. The variables were classified into three groups: the great arteries; the ventricular walls; and the bilateral ventricular cavities. The relationship between the parameters obtained from the CCT images and mean pulmonary arterial pressure (mPAP) was tested and adjusted by the children's body size. Reference curves for the pulmonary trunk diameter (PTD) and ratio of diameter of pulmonary trunk to ascending aorta (rPTAo) of children with CCT images of normal hearts, adjusted for height, were plotted. Threshold lines were established on the reference curves.

Results: PTD and rPTAo on the CCT images were significantly positively correlated with mPAP $(r>0.85, p<0.01)$. Height was the body size parameter most correlated with PTD $(r=0.91, p<0.01)$ and rPTAo $(r=-0.69, p<0.01)$. On the basis of the threshold lines on the reference curves, PTD and rPTAo both showed $88.9 \%$ sensitivity for PAH diagnosis, with negative predictive values of $93.3 \%$ and $92.9 \%$, respectively.

Conclusion: PTD and rPTAo measured from CCT images were significantly correlated with mPAP in children. Reference curves and the formula of PTD and rPTAo adjusted for height could be practical for diagnosing PAH in children.

Keywords: Pulmonary arterial hypertension; Children; Computed tomography

\section{INTRODUCTION}

Pulmonary arterial hypertension (PAH) has a poor prognosis characterized by progressively increasing pulmonary vascular resistance that eventually leads to right ventricular heart failure (1). PAH is diagnosed when

Received October 2, 2018; accepted after revision February 2, 2019.

This study was supported from our National Science Council by a project (NSC 98-2314-B-002-061).

Corresponding author: Ming-Tai Lin, MD, PhD, Department of Pediatrics, National Taiwan University Hospital, No. 7, Chung-Shan South Road, Taipei 100, Taiwan.

- Tel: (886) 2-2312-3456 - Fax: (886) 2-2314-7450

- E-mail: mingtailin@ntu.edu.tw

This is an 0pen Access article distributed under the terms of the Creative Commons Attribution Non-Commercial License (https://creativecommons.org/licenses/by-nc/4.0) which permits unrestricted non-commercial use, distribution, and reproduction in any medium, provided the original work is properly cited. the mean pulmonary arterial pressure (mPAP), assessed through right heart catheterization, exceeds $25 \mathrm{~mm} \mathrm{Hg}$ at rest (2). In the general population, PAH has a low incidence (3). Most children presenting with elevated mPAP have a congenital heart defect (CHD), particularly a prominent chronic left-to-right shunt (4). If the diagnosis is delayed, the prognosis may become poorer and sometimes limit the treatment options (5).

Currently, the major diagnostic evaluation pathway for $\mathrm{PAH}$ involves noninvasive echocardiography followed by cardiac magnetic resonance imaging (MRI) and right heart catheterization (6-9). Transthoracic echocardiography can reveal the morphological and hemodynamic changes in patients with CHD. However, it is limited by its operatordependence and the absence of a suitable acoustic window. Studies have reported widely varying correlations in the relationship between echocardiography-estimated and catheterization-recorded mPAP $(10,11)$. Direct 
measurement of mPAP through cardiac catheterization remains the gold standard examination. Although MRI does not require radiation and is recommended in the United States and Europe, the medical expenses and healthcare systems in Asian countries are completely different from those of Western countries. For example, the relatively limited resources in Asia led to the consensus that cardiac computed tomography (CCT) is appropriate for some cases of CHD (12-14).

Computed tomography (CT) is traditionally used for assessing lung parenchyma and pulmonary vasculature in PAH and identifying or excluding thromboembolic disease, pulmonary artery stenosis, or dilatation $(15,16)$. Advancements in CCT technology have resulted in improved temporal and spatial resolution that can non-invasively provide comprehensive data of the cardiopulmonary status in one examination (17-20). The criteria for evaluating PAH in adults using both conventional non-electrocardiography (ECG)-gated CT and ECG-gated CCT have been reported (2123). However, those guidelines have limitations when used in children $(4,24)$, because the size of physiologically normal cardiovascular structures and the hemodynamics of the pulmonary circulation vary considerably from infancy to adolescence. Regular follow-up of patients to non-invasively estimate their mPAP has become mandatory for monitoring the therapeutic response and ultimately facilitate the advancement of PAH therapy $(25,26)$. Furthermore, the severity of elevated MPAP is a critical indicator for selecting the time of surgery in some CHD cases, such as a chronic unrepaired atrial septal defect (ASD). The purpose of this study was to explore the clinical usefulness of this noninvasive CCT technique for diagnosing and estimating $\mathrm{PAH}$ in children.

\section{MATERIALS AND METHODS}

\section{Patients}

The institutional human research committee approved this retrospective study and waived the need for informed consent. The study protocol conformed to the ethical guidelines of the 1975 Declaration of Helsinki. This study was conducted in a single hospital. The first study period was from October 2004 to September 2015. After obtaining the results of patients during the first study period (steps I-III), additional patient data for the second study period (step IV), from October 2015 to September 2018, were collected for verification. Children with proven pulmonary thromboembolism and underlying diffuse lung parenchyma disorders were excluded. In step I, we enrolled 40 patients with mean age of 16.7 years. In step II, we recruited 115 subjects with mean age of 9.1 years. In step III, we enrolled 78 patients with mean age of 10.6 years. Finally, in step IV, we enrolled 27 patients with mean age of 7.4 years. Patient demographics are presented in Table 1. Cardiac catheterization for mPAP measurement and CCT were performed within 3 months of each other, and no other intervention was performed between the two examinations.

\section{Cardiac CT Acquisition}

Until June 2007, CCT was performed using a 16-slice scanner (LightSpeed 16; GE Healthcare, Milwaukee, WI, USA), followed by a 64-slice scanner (VCT; GE Healthcare) from July 2007 to October 2008, and another 64-slice scanner (SOMATOM Sensation 40; Siemens Healthineers, Erlangen, Germany) from November 2008 onwards. Data were acquired in the caudocranial direction, with a section thickness of $0.625-0.8 \mathrm{~mm}$ without gaps. The matrix size in the $X-Y$ plane was $512 \times 512$ pixels. Nonionic iodinated contrast medium ( $2 \mathrm{~mL} / \mathrm{kg}$ iopromide, Ultravist ${ }^{\circledR} 370$; Bayer AG, Berlin, Germany) was delivered through a power injector to all patients at a flow rate of $0.8-4 \mathrm{~mL} / \mathrm{sec}$ from newborn to adolescence, respectively. The delay time between the start of contrast medium administration and the start of imaging was determined using a bolus-tracking technique. A region in the ascending aorta was used for bolus-tracking; the threshold level was set at $150 \mathrm{HU}$. All cooperative patients were asked to hold their breath during scanning. Quiet respiration without crying was deemed acceptable in younger children and infants who could not comply with this request; therefore, patients aged $<5$ years were routinely sedated with chloral hydrate $(50 \mathrm{mg} /$ $\mathrm{kg}$ ) during examination. The $\mathrm{mAs}$ and $\mathrm{kVp}$ values were ageadjusted in order to reduce the radiation dose, following the as low as reasonably achievable principle $(14,27,28)$. The mean effective radiation doses ranged from $2.0 \mathrm{mSv}$ in newborns to $15.6 \mathrm{mSv}$ in teenagers. This range of radiation doses was lower than that reported in our previous study, although the two studies had 43 overlapping cases (14). Image reconstruction was performed through multi-planar reformatting on an independent workstation. A single reader with 23 years of experience in pediatric CCT assessed the CCT images and obtained the required measurements. The reader was blinded to the mPAP data. 
Table 1. Patient Characteristics

\begin{tabular}{|c|c|c|c|c|c|}
\hline \multirow{2}{*}{ Characteristics } & \multicolumn{2}{|c|}{ Step I $(n=40)$} & \multirow{2}{*}{ Step II $(n=115)$} & \multirow{2}{*}{ Step III $(n=78)$} & \multirow{2}{*}{ Step IV $(n=27)$} \\
\hline & $\mathrm{PAH}(\mathrm{n}=15)$ & No PAH $(n=25)$ & & & \\
\hline Mean age (range) & $\begin{array}{c}17.1 \mathrm{yr} \\
(14.8-18.5 \mathrm{yr})\end{array}$ & $\begin{array}{c}16.4 \mathrm{yr} \\
(13.3-18.3 \mathrm{yr})\end{array}$ & $\begin{array}{c}9.1 \mathrm{yr} \\
(10 \mathrm{~d}-18.9 \mathrm{yr})\end{array}$ & $\begin{array}{c}10.6 \mathrm{yr} \\
(4-18.5 \mathrm{yr})\end{array}$ & $\begin{array}{c}7.4 \mathrm{yr} \\
(9-18.7 \mathrm{yr})\end{array}$ \\
\hline Sex (male, \%) & $5(33.3)$ & $17(68)$ & $78(67.8)$ & $45(57.7)$ & $11(40.7)$ \\
\hline Mean mPAP (mm Hg) (range) & $56.8(25-80)$ & $13.9(6-24)$ & N/A & $26.1(6-94)$ & $26.0(10-58)$ \\
\hline CCT images "Normal" & 8 & 12 & $12^{*}+45$ & $20^{\ddagger}$ & \\
\hline ASD, repaired & 5 & 8 & & $13^{\ddagger}$ & 3 \\
\hline ASD & & & & 13 & 6 \\
\hline VSD, repaired & 2 & 4 & & $6^{\ddagger}$ & \\
\hline VSD & & & & 15 & 3 \\
\hline RA tumor & & 1 & $1^{*}$ & $1^{\ddagger}$ & \\
\hline APVC & & & & 10 & \\
\hline APVC, repaired & & & & & 2 \\
\hline Coronary artery & & & 33 & & 3 \\
\hline Airway & & & 8 & & \\
\hline SVC & & & 7 & & \\
\hline Vegetation & & & 6 & & \\
\hline Pulmonary sequestration & & & 3 & & \\
\hline Others & & & & & $10^{\dagger}$ \\
\hline
\end{tabular}

${ }^{*}$ Cases proved mPAP $<25 \mathrm{~mm} \mathrm{Hg}$ by catheterization from Step I, ${ }^{\top}$ Three suspected primary PAH, 2 hypertrophic cardiomyopathy, 1 patent ductus arteriosus for vascular plug, 1 constrictive pericarditis, 1 restrictive cardiomyopathy, 1 portosystemic shunt and 1 cor triatriatum, ${ }^{\ddagger}$ Cases from Step I. Airway = suspect airway problem, APVC = unrepaired anomalous pulmonary venous connection, ASD = unrepaired atrial septal defect, $\mathrm{CCT}=$ cardiac computed tomography, Coronary = suspect coronary artery problem, $\mathrm{d}=$ day, mPAP = mean pulmonary arterial pressure, N/A = not available, "Normal" = subject's heart in situs solitus, concordant atrioventricular and ventriculoarterial connection, no intracardiac defect, no dilatation of any cardiac chambers, and no vascular anomaly, PAH = pulmonary arterial hypertension, RA = right atrium, SVC = suspect superior vena cava problem, Vegetation = small intracardiac vegetation, VSD = unrepaired ventricular septal defect, $\mathrm{yr}=$ year

\section{Step I: Analysis of the Relationship Between CCT Measurements and mPAP}

An elevation in mPAP leads to progressive dilatation of the pulmonary trunk, bilateral pulmonary arteries, and right ventricle (RV) as well as RV hypertrophy $(29,30)$. As the RV pressure approaches that of the left ventricle (LV), the LV becomes compressed and exhibits increased pressure, causing LV hypertrophy (31). Clinically, we can also observe the dilatation of the inferior vena cava (IVC) during passive congestion due to PAH. Studies have reported the pulmonary trunk diameter (PTD) and PTD-to-ascending aorta diameter ratio ( $\mathrm{rPTAo}$ ) on CCT images to be positive predictors of underlying PAH in adults $(22,32)$. In step I, we tried to explore more indicators other than PTD and rPTAo in children (Fig. 1). The legend in Figure 1 provides a more comprehensive description of the measurements we performed.

Obtaining both the CCT and cardiac catheterization data of an individual with an absolutely normal heart is difficult, except in volunteers. The indications of this step I period for CCT were used to identify any unusual pathology limited by or uncertain in echocardiography in patients who had suspected PAH on the basis of clinical presentation. Therefore, patients with "normal" cardiac anatomy on CCT images with or without a final diagnosis of PAH were selected. The so-called "normal" cardiac anatomy on CCT images is defined by situs solitus, concordant atrioventricular and ventriculoarterial connection, no intracardiac defect, and no anatomical vascular anomaly at the great vessels connecting to the heart. These subjects received both CCT and right heart catheterization.

\section{Step II: Construction of Reference Curves for CCT-Image} Parameters Adjusted for Body Size

Children with complete normal cardiac anatomy rarely undergo cardiac catheterization for mPAP measurement. Thus, selection of individuals with a normal heart and an $\mathrm{mPAP}<25 \mathrm{~mm} \mathrm{Hg}$ determined through right cardiac catheterization may yield limited cases. Consequently, in this step II period, the inclusion criteria were extended to 


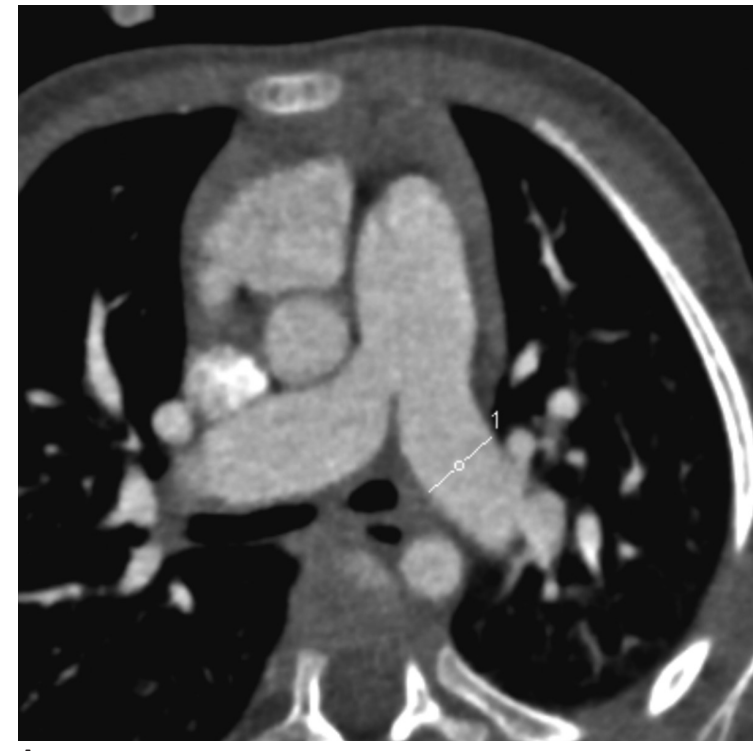

A

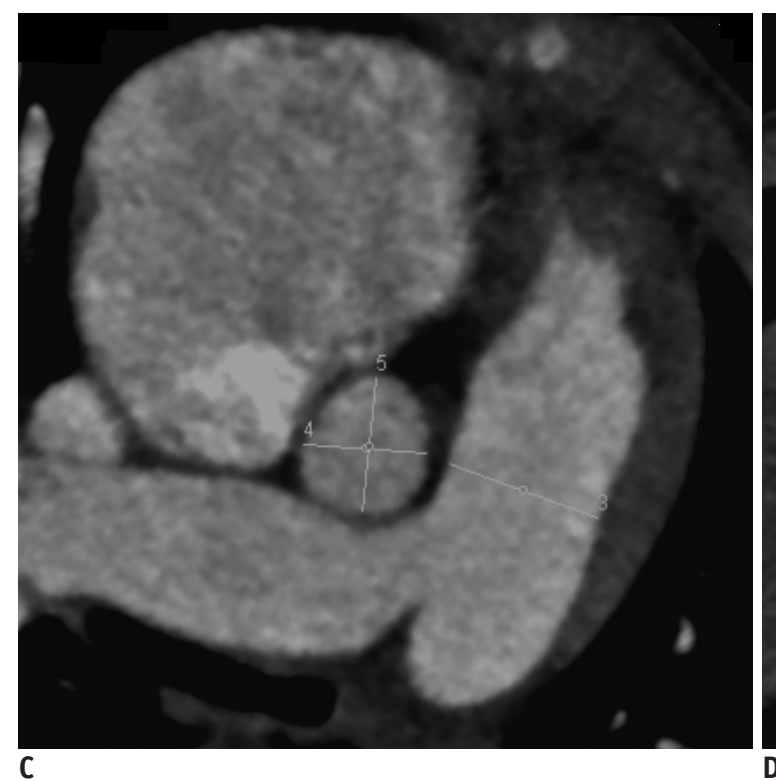

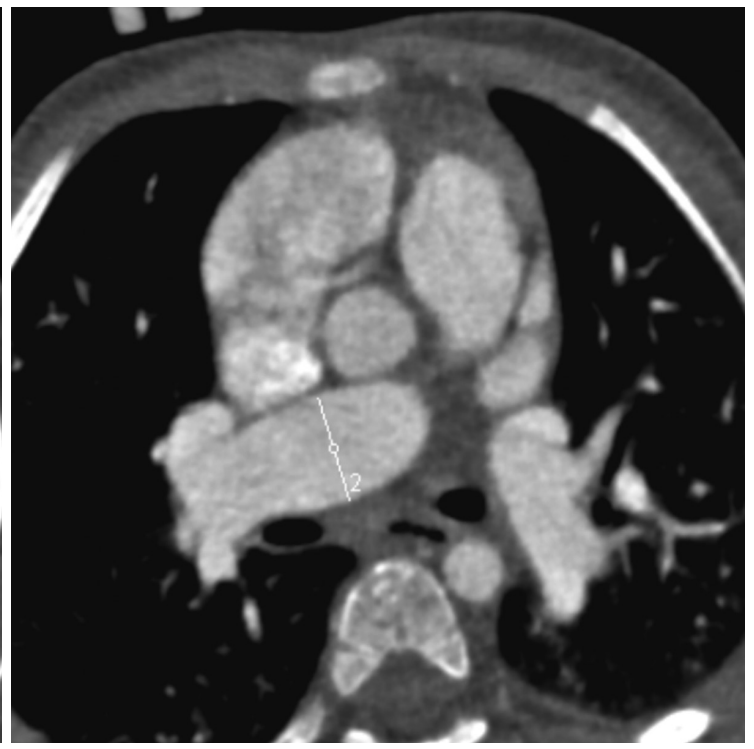

B

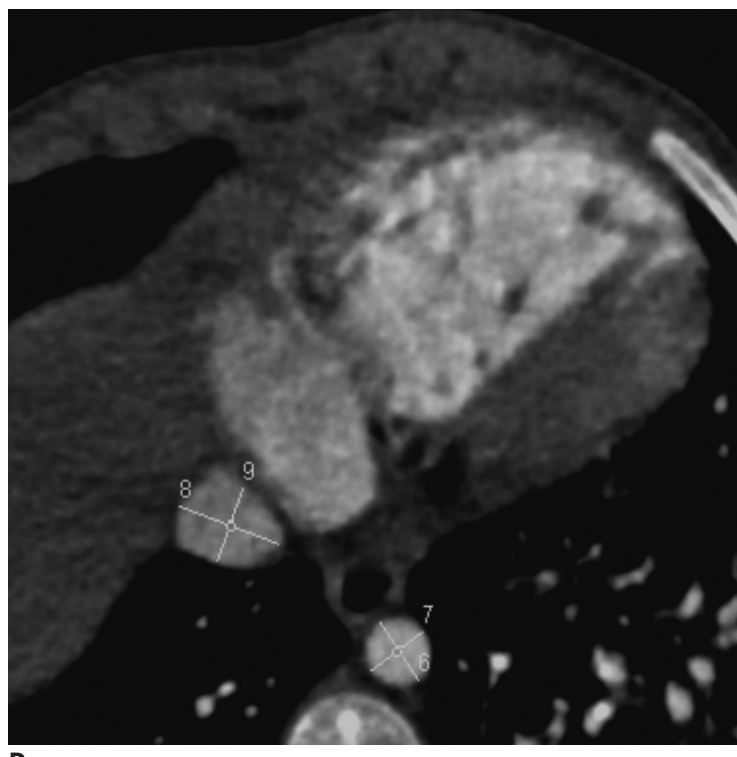

D

Fig. 1. Parameters measured on end-diastole phase of contrast-enhanced CCT images to diagnose pulmonary arterial hypertension.

A, B, D. Images were obtained in transverse planes. C. Image was obtained in tilted oblique axial plane to reveal whole pulmonary trunk from pulmonary annulus to bifurcation. E, F. Images were at middle ventricular level with image (E) on cardiac short-axis plane and image (F) on cardiac four-chamber plane. Twenty measurements were performed and annotated 1-20, and all of these measurements could also be easily performed on picture archiving and communication system. 1 = maximal diameter of left pulmonary artery before branching, $2=$ maximal diameter of right pulmonary artery before branching, $3=$ maximal diameter of middle pulmonary trunk before bifurcation, 4 and $5=$ perpendicular diameters of ascending aorta on same image that measured pulmonary trunk, 6 and 7 = perpendicular diameters of descending aorta at level through diaphragm, 8 and $9=$ perpendicular diameters of inferior vena cava on same image that measured descending aorta, 10-12=middle ventricular myocardial thickness of RV, septum, and LV respectively, 13 and 14, 15 and $16=$ perpendicular height and width intersects at midpoints of RV and LV, respectively, 17 and 18, 19 and $20=$ perpendicular length and width intersects at midpoints of RV and LV respectively, 21 and 23,22 and $24=$ area of LV and RV respectively. CCT = cardiac computed tomography, LV = left ventricle, RV = right ventricle

subjects with minor cardiovascular or bronchopulmonary problems who had no clinical presentation of any cardiovascular symptoms and signs. These CCT indications in this step II period ranged from checking for suspected coronary artery abnormalities, small intracardiac vegetations, any incidental shadows on echocardiograms or suspected bronchopulmonary anomalies that were finally cleared as "normal" cardiac anatomy, or anomalies that were not related to the target parameters we measured.

To reduce time, only the representative parameters 

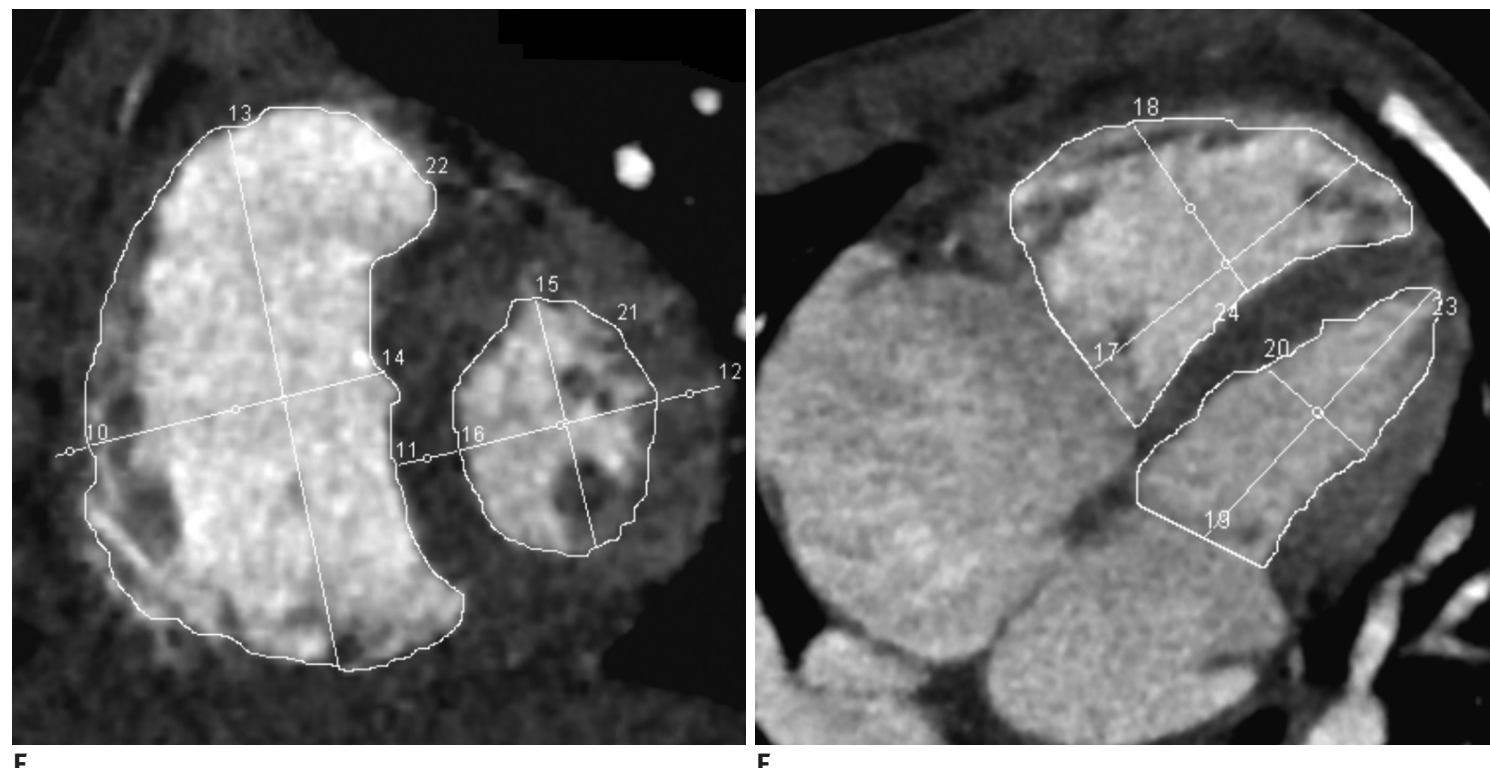

Fig. 1. Parameters measured on end-diastole phase of contrast-enhanced CCT images to diagnose pulmonary arterial hypertension. A, B, D. Images were obtained in transverse planes. C. Image was obtained in tilted oblique axial plane to reveal whole pulmonary trunk from pulmonary annulus to bifurcation. E, F. Images were at middle ventricular level with image (E) on cardiac short-axis plane and image (F) on cardiac four-chamber plane. Twenty measurements were performed and annotated 1-20, and all of these measurements could also be easily performed on picture archiving and communication system. $1=$ maximal diameter of left pulmonary artery before branching, $2=$ maximal diameter of right pulmonary artery before branching, $3=$ maximal diameter of middle pulmonary trunk before bifurcation, 4 and $5=$ perpendicular diameters of ascending aorta on same image that measured pulmonary trunk, 6 and $7=$ perpendicular diameters of descending aorta at level through diaphragm, 8 and $9=$ perpendicular diameters of inferior vena cava on same image that measured descending aorta, $10-12=$ middle ventricular myocardial thickness of RV, septum, and LV respectively, 13 and 14,15 and $16=$ perpendicular height and width intersects at midpoints of RV and LV, respectively, 17 and 18, 19 and $20=$ perpendicular length and width intersects at midpoints of RV and LV respectively, 21 and 23,22 and $24=$ area of $L V$ and RV respectively. $C C T=$ cardiac computed tomography, LV = left ventricle, RV = right ventricle

from step I were measured. In this step II, by using the CCT-image parameters strongly correlated with the mPAP obtained in step I, we constructed "normal" reference curves of CCT images. To apply these reference curves to children, we adjusted the parameters according to their highest correlated body sizes, which varied widely.

\section{Step III: Establishing Diagnostic Criteria for PAH and Prediction Formulas for mPAP Adjusted for Body Size}

In the step III period, by using the representative CCTimage parameters that were strongly correlated with mPAP and the most representative parameters for body size obtained in step II, we created formulas to predict mPAP in children. By using these formulas, we could set a threshold line on the "normal" reference curves constructed in step II to enable the diagnosis of PAH in children.

The number of cases included in our first study period in step I was insufficient. Therefore, we extended our inclusion criteria to also include patients with one of the different types of small ASDs, small ventricular septal defects, and partial anomalous pulmonary venous connections. The term "small" was not used to describe small size in the structure but to suggest that the shunting was limited hemodynamically. Usually, we could estimate the pulmonary (Qp) and aortic (Qs) flow from the velocity time integrals on echocardiography. If $Q p / Q$ s was less than 1.5, the defect was thought to be small. The reason for extending the inclusion criteria in this manner was because the primary pathologies were far away from the measured PTD and rPTAo, as reported in the literature (22, 32). The indications for CCT of these added subjects in this step III were to check for the presence of any associated anomalies, such as coronary artery or airway anomalies, hidden in echocardiography, which was finally cleared. Right heart catheterizations were indicated in these additional patients for verifying cardiovascular anomalies found with echocardiography and demonstrating their hemodynamic significance for the decision to perform surgery on them.

\section{Step IV: Verification}

In step IV, patients who had underlying cardiovascular anomalies directly related to the analyzed results from 
the preceding steps were excluded. All these patients were different from the patients of the previous steps I, II, and III. Indications for CCT were to check for coronary pathology, cardiovascular anomaly, and myocardial and pericardial abnormalities. By using the eligible patient data from CCT and right heart catheterization, we verified the proposed threshold lines and formulas from the preceding steps.

\section{Statistical Analysis}

Descriptive statistics and the Student's $t$ test were used to compare the mean measurements of the study and control groups. Relationships between parameters were checked by using 2-tailed Pearson correlation. Correlation coefficient $(r)$ values $>0.8$ were considered significant. Reference curves were plotted through general linear regression analysis according to the highest coefficient of determination $\left(R^{2}\right)$ values obtained in curve-fitting. Statistical analyses were performed using the commercially available software EXCEL (version 15, Microsoft, Redmond, WA, USA) and SPSS (version 16, SPSS Inc., Chicago, IL, USA). A formula for predicting mPAP by using the most correlated CCT-image parameters, with adjustments for the most correlated body size parameter of children, was constructed through surface regression (Centurion XVII, StatGraphics, Warrenton, VA, USA). $P<0.05$ was considered significant.

\section{RESULTS}

\section{Step I: Correlation of PTD and rPTAo with mPAP}

CCT was used to check any subtle abnormality in the heart, thromboembolism, and lung parenchymal pathology that finally cleared as normal cardiopulmonary anatomy. The difference in the age distribution between the PAH and non-PAH groups was not significant $(p=0.28)$, whereas mPAP values differed significantly between these groups ( $p$ $<0.01$ ). The parameters on the CCT images that correlated the most with mPAP were the PTD and rPTAo. The remaining 25 CCT parameters showed limited correlation with mPAP (Table 2).

\section{Step II: Normal Reference Curves of PTD and rPTAo Adjusted for Height in Children}

Height was the independent variable that correlated the most with both PTD and rPTAo $(r=0.91$ and -0.69 respectively; both $p<0.01)$. Other variables of children's body size had weaker correlations than height. The
Table 2. Correlation between Mean Pulmonary Artery Pressure and Parameters Measured on CCT

\begin{tabular}{|c|c|c|}
\hline Parameters & r Valve & $P^{*}$ \\
\hline \multicolumn{3}{|l|}{ Great arteries } \\
\hline CT-McGoon & 0.69 & $<0.001$ \\
\hline PTD & 0.89 & $<0.001$ \\
\hline rPTAo & 0.86 & $<0.001$ \\
\hline AAo & 0.14 & 0.458 \\
\hline IVC & 0.51 & 0.001 \\
\hline DAo & -0.19 & 0.381 \\
\hline IVC/DAo ratio & 0.50 & $<0.001$ \\
\hline \multicolumn{3}{|l|}{ Myocardial wall thickness } \\
\hline RV & 0.41 & 0.004 \\
\hline $\mathrm{RV} /$ septum ratio & 0.58 & $<0.001$ \\
\hline Septum & -0.34 & 0.018 \\
\hline $\mathrm{LV} /$ septum ratio & 0.31 & 0.022 \\
\hline LV & 0.07 & 0.874 \\
\hline $\mathrm{RV} / \mathrm{LV}$ ratio & 0.40 & 0.013 \\
\hline \multicolumn{3}{|c|}{$\begin{array}{l}\text { Size of ventricles at mid-ventricular } \\
\text { level on short-axis view }\end{array}$} \\
\hline RV height & 0.40 & 0.014 \\
\hline RV area & 0.49 & 0.001 \\
\hline RV width & 0.51 & 0.001 \\
\hline Area RV/LV ratio & 0.60 & $<0.001$ \\
\hline LV height & -0.30 & 0.088 \\
\hline LV area & -0.52 & $<0.001$ \\
\hline LV width & -0.50 & 0.001 \\
\hline \multicolumn{3}{|c|}{$\begin{array}{l}\text { Size of ventricles at mid-ventricular } \\
\text { level on four-chamber view }\end{array}$} \\
\hline RV length & 0.52 & 0.001 \\
\hline RV area & 0.48 & 0.001 \\
\hline RV width & 0.40 & 0.014 \\
\hline Area RV/LV ratio & 0.59 & $<0.001$ \\
\hline LV length & -0.28 & 0.120 \\
\hline LV area & -0.53 & $<0.001$ \\
\hline LV width & -0.55 & $<0.001$ \\
\hline
\end{tabular}

* Significance of Pearson correlation test by 2-tailed test. $\mathrm{AAo}=$ ascending aorta diameter, CT-McGoon = sum of diameters of left and right pulmonary arteries divided by diameter of descending aorta, Dao = descending aorta diameter, IVC = inferior vena cava diameter, IVC/Dao = ratio of diameter of IVC to DAo, LV = left ventricle, PTD = pulmonary trunk diameter, $r$ PTAo $=$ ratio of diameter of pulmonary trunk to ascending aorta, RV = right ventricle

correlation coefficients to PTD were 0.82 for age, 0.82 for body surface area (BSA), and 0.75 for weight, respectively. The correlation coefficients to rPTAo were -0.61 for age, -0.56 for BSA, and -0.5 for weight, respectively. By using height as a single key independent variable, we established the normal reference curves of PTD and rPTAo, adjusted for variable body size in children. By using general linear 


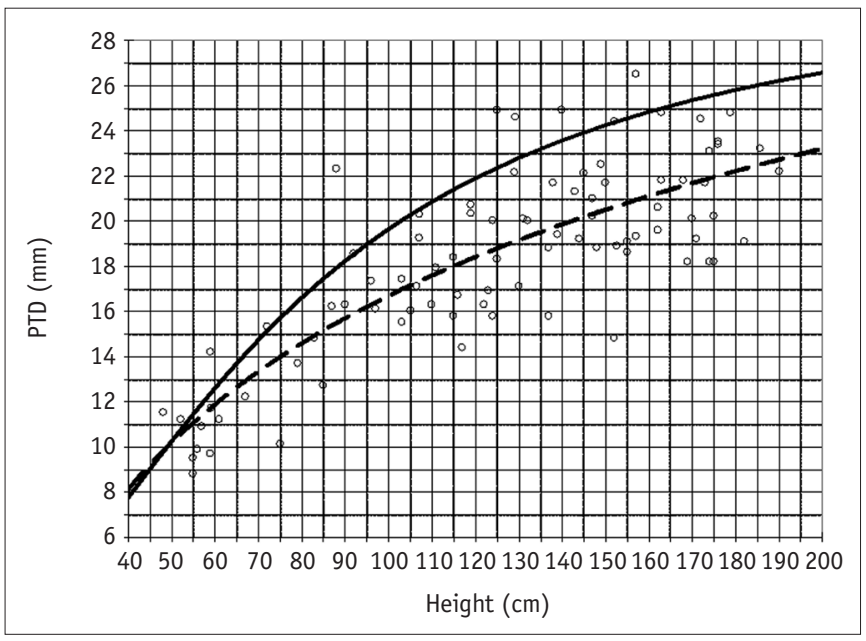

A

Fig. 2. Relationship of PTD (A) and rPTAo (B) to height in children determined using CCT images of normal hearts. Dashes represent best-fitting linear regression of mean of study subjects from our study step II. Solid lines indicate thresholds of 25-mm $\mathrm{Hg}$ mean pulmonary arterial pressure calculated from subjects from our study step III. Subjects from steps II and III were not same. PTD = pulmonary trunk diameter, rPTAo $=$ PTD-to-ascending aorta diameter ratio

regression analysis on the data with the best-fitting algorithm, we obtained a height versus PTD curve through quadratic curve estimation (PTD $=1.05635 \times$ [height ${ }^{0.59132}$ ], $\left.\mathrm{R}^{2}=0.71, p<0.001\right)($ Fig. $2 \mathrm{~A}$ ) and a height versus rPTAo curve through linear estimation $(\mathrm{rPTAo}=-0.002134 \mathrm{x}$ height $\left.+1.343391, \mathrm{R}^{2}=0.49, p<0.001\right)$ (Fig. 2B).

\section{Step III: Formulas to Estimate mPAP and Establish} Thresholds on Reference Curves

However, height data were available for only 72 patients. We performed surface regression analysis to obtain the best-fitting surfaces and formulas to estimate mPAP (in mm $\mathrm{Hg}$ ) by using PTD (in $\mathrm{cm}$ ) or rPTAo which both took height (in $\mathrm{cm}$ ) of children into consideration (Supplementary Fig. 1 in the online-only Data Supplement).

The resulting formulas to estimate mPAP were as follows:

$$
\begin{array}{r}
m P A P=-54.83-12021.1 \times\left(\text { height }^{-1.78}\right)+6.98 \times\left(\mathrm{PTD}^{0.71}\right) \\
+11080 \times\left(\text { height }^{-1.78}\right) \times\left(\mathrm{PTD}^{0.71}\right) \\
\quad\left[\mathrm{R}^{2}=0.68, p<0.001\right] \\
\mathrm{mPAP}=-0.59-1.61^{-10} \times\left(\text { height }^{5}\right)+16.49 \times\left(\mathrm{rPTA}^{1.18}\right)+ \\
1.77^{-10} \times\left(\text { height }^{5}\right) \times\left(\mathrm{rPTAo}^{1.18}\right) \quad \\
\quad\left[\mathrm{R}^{2}=0.58, p<0.001\right]
\end{array}
$$

These formulas were used to determine the value of PTD or rPTAo at different heights that had an estimated mPAP of $25 \mathrm{~mm} \mathrm{Hg}$. Thereafter, we could arbitrarily set solid threshold lines on the reference curves (Fig. 2). Thus, PAH could be straightforwardly diagnosed in patients in whom
CCT parameters lay above the threshold lines, provided that their height was known. The formula could be written into a computer program for calculation in daily practice.

\section{Step IV: Good Sensitivity and Negative Prediction}

The CCT-image parameters we used in the first study period that predict mPAP were PTD and rPTAo. Therefore, in this step, we excluded patients with native pathologies at the great arteries (e.g., tetralogy of Fallot, transposition of the great arteries and pulmonary atresia, etc.) and patients who had undergone surgery directly on the great arteries.

From these reference curves, we could diagnose PAH in patients with parameters lying above the solid threshold lines. The sensitivity, specificity, positive predictive value, negative predictive value, and accuracy of the threshold line of height versus PTD were $88.9 \%, 77.8 \%, 66.7 \%$, $93.3 \%$, and $81.5 \%$, respectively. The sensitivity, specificity, positive predictive value, negative predictive value, and accuracy of the threshold line of height versus rPTAo were $88.9 \%, 72.2 \%, 61.5 \%, 92.9 \%$, and $77.8 \%$, respectively (Supplementary Fig. 2 in the online-only Data Supplement).

By using our formulas, derived from step III, we estimated mPAP using PTD and height or rPTAo and height. Values of mPAP estimated using both formulas showed no significant difference $(p<0.05)$.

\section{DISCUSSION}

In this study, PTD and rPTAo were found to be the two 
most critical parameters on the CCT images and were strongly and significantly correlated with mPAP in children. This is consistent with previous findings for adults (2123). The parameters measured from the CCT images, other than PTD and rPTAo, exhibited only limited correlations. Thus, the initial proposed parameters at the ventricular or myocardial levels are less significant in predicting mPAP in children. It is possible that there are more complex and remote mechanisms of their structural remodeling in response to the elevated mPAP. For example, IVC size could be confounded by the presence or absence of tricuspid regurgitation. Hence, for clinical use, only PTD and rPTAo were considered the most practical parameters for PAH diagnosis. However, because the body size of pediatric patients varies widely from infancy to young adulthood, using a single cutoff point to differentiate between patients with and without PAH is unreasonable. In this study, we demonstrated that the patient's height is the most strongly correlated parameter that can be used to adjust for body size in pediatric populations. In fact, the most right-hand end of our threshold lines had individual height similar to an adult's, so our results also could be applied in adults. These two solid threshold lines have been confirmed to have good sensitivity and good negative predictive value for the diagnosis of PAH. Moreover, because of substantial advancements in PAH medication, regular follow-up after treatment is necessary. Our formulas can be used to estimate MPAP and may be suitable for noninvasive followup of outpatients to monitor their therapeutic response.

There were some limitations in our study. The studied populations in steps I-III were not identical. This resulted in some discrepant data points on Figure 2A. The possible elevated mPAP values in step II subjects have not been measured through catheterization. Our calculated solid threshold line for the height versus PTD curve crossed with the regression line at early infancy. The reason for this is unclear. Because of the small sample size of our study, studies using a larger population are warranted to obtain more suitable correlations, curves, regression fitting, and verification. The curves and formula suggested in this study cannot be used in patients with substantial pulmonary regurgitation, such as absent pulmonary valve syndrome and repaired tetralogy of Fallot, because their pathophysiology is not the same as in our study population. Similarly, valvular stenosis either on the aortic or pulmonary artery leads to post-stenotic dilation of the artery, so they are not suitable to be applied with our results. A single observer measured the CCT-image parameters; nevertheless, it has been reported that the level of intra- and inter-observer correlation is good in such measurements $(22,33,34)$. Radiation exposure is an inherent disadvantage of CCT. However, with advances in $\mathrm{CT}$ technologies, $\mathrm{CT}$ radiation dose is being reduced markedly, and the dose of modern CCT was lower than that of diagnostic cardiac catheterization $(14,27,28)$.

In conclusion, PTD and rPTAo on the CCT images are significantly correlated with mPAP in children. We found that the proposed thresholds of rPTAo and PTD measured on CCT have very good negative predictive value. The curves and formula obtained in this study may facilitate the diagnosis of PAH in children.

\section{Supplementary Materials}

The online-only Data Supplement is available with this article at https://doi.org/10.3348/kjr.2018.0673.

\section{Conflicts of Interest}

The authors have no potential conflicts of interest to disclose.

\section{ORCID iDs}

Ming-Tai Lin

https://orcid.org/0000-0002-1718-7137

Shyh-Jye Chen

https://orcid.org/0000-0001-6181-011X

\section{REFERENCES}

1. Humbert M, Sitbon O, Yaïci A, Montani D, O'Callaghan DS, Jaïs $X$, et al.; French Pulmonary Arterial Hypertension Network. Survival in incident and prevalent cohorts of patients with pulmonary arterial hypertension. Eur Respir J 2010;36:549-555

2. Fowler NO, Westcott RN, Scott RC. Normal pressure in right heart and pulmonary artery. Am Heart J 1953;46:264-267

3. Peacock AJ, Murphy NF, McMurray JJ, Caballero L, Stewart S. An epidemiological study of pulmonary arterial hypertension. Eur Respir J 2007;30:104-109

4. Gupta SK, Saxena A, Gulati GS. Evaluation of pulmonary hypertension in a child: role of computed tomography. Indian J Pediatr 2011;78:1417-1419

5. Nauser TD, Stites SW. Diagnosis and treatment of pulmonary hypertension. Am Fam Physician 2001;63:1789-1798

6. Celermajer DS, Marwick T. Echocardiographic and right heart catheterization techniques in patients with pulmonary arterial hypertension. Int J Cardiol 2008;125:294-303 
7. Schannwell CM, Steiner S, Strauer BE. Diagnostics in pulmonary hypertension. J Physiol Pharmacol 2007;58 Suppl 5(Pt 2):591-602

8. Hegewald MJ, Markewitz B, Elliott CG. Pulmonary hypertension: clinical manifestations, classification and diagnosis. Int J Clin Pract Suppl 2007;(156):5-14

9. McLaughlin VV, McGoon MD. Primary pulmonary hypertension. Hospital Physician Board Review Manual 2002;9:1-11

10. Bossone E, Duong-Wagner TH, Paciocco G, Oral H, Ricciardi M, Bach DS, et al. Echocardiographic features of primary pulmonary hypertension. J Am Soc Echocardiogr 1999;12:655662

11. McGoon M, Gutterman D, Steen V, Barst R, McCrory DC, Fortin TA, et al. Screening, early detection, and diagnosis of pulmonary arterial hypertension: ACCP evidence-based clinical practice guidelines. Chest 2004;126(1 Suppl):14S-34S

12. ASCI CCT \& CMR Guideline Working Group, Tsai IC, Choi BW, Chan C, Jinzaki M, Kitagawa K, et al. ASCI 2010 appropriateness criteria for cardiac computed tomography: a report of the Asian Society of Cardiovascular Imaging Cardiac Computed Tomography and Cardiac Magnetic Resonance Imaging Guideline Working Group. Int J Cardiovasc Imaging 2010;26 Suppl 1:1-15

13. Tsai IC, Goo HW. Cardiac CT and MRI for congenital heart disease in Asian countries: recent trends in publication based on a scientific database. Int J Cardiovasc Imaging 2013;29 Suppl 1:1-5

14. Yang JC, Lin MT, Jaw FS, Chen SJ, Wang JK, Shih TT, et al. Trends in the utilization of computed tomography and cardiac catheterization among children with congenital heart disease. J Formos Med Assoc 2015;114:1061-1068

15. Kuriyama K, Gamsu G, Stern RG, Cann CE, Herfkens RJ, Brundage BH. CT-determined pulmonary artery diameters in predicting pulmonary hypertension. Invest Radiol 1984;19:1622

16. Ley S, Kreitner KF, Fink C, Heussel CP, Borst MM, Kauczor HU. Assessment of pulmonary hypertension by $\mathrm{CT}$ and MR imaging. Eur Radiol 2004;14:359-368

17. Di Guglielmo L, Dore R, Vespro V. Pulmonary hypertension: role of computed tomography and magnetic resonance imaging. Ital Heart J 2005;6:846-851

18. McLure $L E$, Peacock $A J$. Imaging of the heart in pulmonary hypertension. Int J Clin Pract 2007;61(S156):15-26

19. Chen BB, Chen SJ, Wu MH, Li YW, Lue HC. EBCT-McGoon ratio a reliable and useful method to predict pulmonary blood flow non-invasively. Chinese J Radiol 2007;32:1-8

20. Goo HW. State-of-the-art CT imaging techniques for congenital heart disease. Korean J Radiol 2010;11:4-18

21. Corson N, Armato SG 3rd, Labby ZE, Straus C, Starkey A, Gomberg-Maitland M. CT-based pulmonary artery measurements for the assessment of pulmonary hypertension.
Acad Radiol 2014;21:523-530

22. Ng CS, Wells AU, Padley SP. A CT sign of chronic pulmonary arterial hypertension: the ratio of main pulmonary artery to aortic diameter. J Thorac Imaging 1999;14:270-278

23. Haimovici JB, Trotman-Dickenson B, Halpern EF, Dec GW, Ginns LC, Shepard JA, et al. Relationship between pulmonary artery diameter at computed tomography and pulmonary artery pressures at right-sided heart catheterization. Massachusetts General Hospital lung transplantation program. Acad Radiol 1997;4:327-334

24. Mullen MP. Diagnostic strategies for acute presentation of pulmonary hypertension in children: particular focus on use of echocardiography, cardiac catheterization, magnetic resonance imaging, chest computed tomography, and lung biopsy. Pediatr Crit Care Med 2010;11(2 Suppl):S23-S26

25. Lee YH, Song GG. Meta-analysis of randomized controlled trials of bosentan for treatment of pulmonary arterial hypertension. Korean J Intern Med 2013;28:701-707

26. Wang RC, Jiang FM, Zheng QL, Li CT, Peng XY, He CY, et al. Efficacy and safety of sildenafil treatment in pulmonary arterial hypertension: a systematic review. Respir Med 2014;108:531537

27. Jin KN, Park EA, Shin CI, Lee W, Chung JW, Park JH. Retrospective versus prospective ECG-gated dual-source CT in pediatric patients with congenital heart diseases: comparison of image quality and radiation dose. Int $\mathrm{J}$ Cardiovasc Imaging 2010;26 Suppl 1:63-73

28. Goo HW. CT radiation dose optimization and estimation: an update for radiologists. Korean J Radiol 2012;13:1-11

29. Remy-Jardin M, Delhaye D, Teisseire A, Hossein-Foucher C, Duhamel A, Remy J. MDCT of right ventricular function: impact of methodologic approach in estimation of right ventricular ejection fraction, part 2. Am J Roentgenol 2006;187:1605-1609

30. Savino G, Zwerner P, Herzog C, Politi M, Bonomo L, Costello $\mathrm{P}$, et al. CT of cardiac function. J Thorac Imaging 2007;22:86100

31. Nakamura K, Miyahara Y, Ikeda S, Naito T. Assessment of right ventricular diastolic function by pulsed Doppler echocardiography in chronic pulmonary disease and pulmonary thromboembolism. Respiration 1995;62:237-243

32. Bugnone AN, Viamonte M Jr, Garcia H. Imaging findings in human immunodeficiency virus-related pulmonary hypertension: report of five cases and review of the literature. Radiology 2002;223:820-827

33. Edwards PD, Bull RK, Coulden R. CT measurement of main pulmonary artery diameter. Br J Radiol 1998;71:1018-1020

34. Devaraj A, Well AU, Meister MG, Corte TJ, Wort SJ, Hansell DM. Detection of pulmonary hypertension with multidetector CT and echocardiography alone and in combination. Radiology 2010;254:609-616 